\title{
ANTIOXIDANT AND ANTI-DIABETIC EFFECTS OF AURICULARIA AURICULAR POLYSACCHARIDES AND THEIR DEGRADATION BY ARTIFICIAL GASTROINTESTINAL DIGESTION - BIOACTIVITY OF AURICULARIA AURICULAR POLYSACCHARIDES AND THEIR HYDROLYSATES
}

\author{
Aoxue Lu, Mengen Yu, Meng Shen, Zhiyu Fang, Yaoyao Xu, Shuang Wang, \\ Yongjun Zhang ${ }^{\bowtie}$, Weimin Wang \\ College of Life Sciences, China Jiliang University \\ Xueyuan Street, Xiasha, Hangzhou, Zhejiang, 310018, China
}

\begin{abstract}
Background. Auricularia auricular polysaccharides (AAPs) derived from the dried fruit body of A. auricular are valuable compounds with many bioactivities. This research aimed to investigate the antioxidant and antidiabetic activities of these polysaccharides and their artificial gastrointestinal fluid hydrolysates (AAPHs). Material and methods. Artificially simulated gastrointestinal fluid was used to obtain polysaccharide-derived fragments, and a rat model of type 2 diabetes mellitus (T2DM) using a high-fat diet and low-dose streptozotocin (STZ) was established to assess their antioxidant and anti-diabetic effects.

Results. It was found that AAPs and AAPHs were both heteropolysaccharides and were comprised of arabinose, xylose, mannose, 2-deoxy-glucose, glucose and glucosamine, but at different mole ratios. AAPHs was purified by Sephadex G-100 chromatography to produce three fractions, namely, AAPHs1, AAPHs2, and AAPHs 3 . The molecular weights of these three fractions were 320,169 , and $62 \mathrm{kDa}$ respectively. Both AAPs and AAPHs exhibited the evident ability to enhance the activities of antioxidant enzymes and the level of GSH, while increasing the content of liver glycogen and plasma C-peptide compared with the diabetic model group $(p<0.05)$. Furthermore, AAPHs could cause a marked improvement in glucose-stimulated GLP-1 secretion from $0 \mathrm{~min}$ to $30 \mathrm{~min}(p<0.05)$.

Conclusions. The possible mechanism was that AAPHs could partly restore the STZ-induced impairment of GLP-1 secretion, and inhibit the oxidative stress pathway, and thereby alleviate the progression of diabetes. This data demonstrated that the molecular mole ratio and molecular weight had a definite effect on antioxidant and anti-diabetic activities.
\end{abstract}

Keywords: Auricularia auricular polysaccharide, simulated hydrolysates, antioxidant effect, anti-diabetic activity, T2DM rats

\footnotetext{
This study was supported by the National Natural Science Foundation of China $(31871779,31371765)$ and National Undergraduate Innovation and Entrepreneurship Training Program (201610356016).
} 


\section{INTRODUCTION}

T2DM is a common metabolic disorder which is a great public health concern, with the number of cases increasing across the world (Chapman et al., 2017). Recent assessments from the International Diabetes Federation (IDF) show that older adults ( $\geq 65$ years) account for approximately $25 \%$ of DM cases globally (Chapman et al., 2017). The modern treatment for T2DM is mainly via Western medicine with a series of synthetic agents. However, the severe side-effects of medications make complementary and alternative therapies more appealing for many patients and clinicians (Liu et al., 2016), and a diet-based approach to delay the need for such anti-diabetic medication would be a useful adjunct (Lau, 2014).

Auricularia auricular, a widely distributed nontoxic edible mushroom in Asia, is frequently consumed as a culinary-medicinal mushroom (Qiu et al., 2016). A. auricula has been consumed in traditional medicine as recently as the 19th century. The fruit bodies are wood ears or jelly ears with a high carbohydrate content (Zhang et al., 2011). A. auricular polysaccharides (AAPs) are abundant in fruit bodies, which have been reported to show a large variety of physiological activities, including antioxidant activity (Xu et al., 2016; Zhang et al., 2011), immuno-enhancing properties (Nguyen et al., 2012), anti-tumor activity (Mizuno et al., 1995), the attenuation of the inflammatory response, oxidative stress and lipid deposition (Chiu et al., 2014), anticoagulant activity (Yoon et al., 2003), and hypoglycemic effects (Kim et al., 2007; Yuan, 1998), and have attracted much attention.

The biological and physicochemical properties of biomacromolecules are different from those of conventional drug substances, particularly with regard to their molecular weight, physicochemical stability, solubility, biological half-life, conformational stability, oral bioavailability, dose requirements, and administration (Wang et al., 2009; Xiong, 2016). Natural polysaccharides exhibiting their biological activities are concerned with the oligosaccharide derivatives that are produced by gastric acid or gastric enzymes in the digestive tract (Agbagla-Dohnani et al., 2012; Wang et al., 2016). Several studies in recent years have shown that the derived oligosaccharides produced by the acid or enzymatic hydrolysis of polysaccharides can be used as antioxidants or prebiotics (Daisy et al., 2015; Suphavadee et al., 2016; Xu et al., 2015). Numerous experimental studies have been carried out to evaluate the bioactivities of A. auricular polysaccharides and their derivatives. However, to date, little research has been carried out on the potential antioxidant and anti-diabetic effects of the polysaccharide hydrolysates obtained from this mushroom. The aims of this study were to prepare the artificial gastrointestinal fluid simulated hydrolysate (AAPHs) from A. auricula polysaccharides (AAPs) and to assess the antioxidant and anti-diabetic potential of AAPs and AAPHs using a rat model with diabetes induced by a high-fat diet and low-dose STZ, then to analyze the effect of gastric acid and enzymes on the biological activity of AAPs.

\section{MATERIALS AND METHODS}

\section{Materials}

Auricularia auricula was collected from a farmer's market (Greater Khingan Mountains, Heilongjiang, China). The materials were washed and dried in a hot air oven (DHG-9023AD, China) at $70^{\circ} \mathrm{C}$, then pulverized to a particle diameter size of $100-150 \mu \mathrm{m}$.

Acarbose (biologically pure), was purchased from Nanjing Duly Biotech Co., Ltd (Nanjing, Jiangsu Province, China). STZ, $\alpha$-amylase, pepsin (1:10 000) and pancreatin (1:4000) were obtained from Sigma Chemical Co. (St. Louis, MO, USA). Detection kits for the following were provided by Nanjing Jiancheng Bioengineering Institute (Nanjing, China): glutathione peroxidase (GSH-PX), malonaldehyde (MDA), reduced glutathione (GSH), glutathione reductase (GR) and catalase (CAT). ELISA test kits for insulin and C-peptide were purchased from Shanghai Qiyi Biotechnology Company (Shanghai, China). A one-touch glucometer (for the analysis of blood glucose) was provided by Roche Diagnostics GmbH (Mannheim, Germany). All other chemicals were of analytical grade.

\section{Preparation of AAPs and AAPHs}

Preparation of AAPs. AAPs were prepared by extraction from A. auricular as previously described (Xu et al., 2016). Briefly, soluble components of $A$. auricula 
powders were extracted with 100 volumes of $1 \%(\mathrm{w} / \mathrm{v})$ $\mathrm{NaOH}$ at $80^{\circ} \mathrm{C}$, followed by neutralization with hydrochloric acid, then centrifuged at $8000 \mathrm{rpm}$ for $20 \mathrm{~min}$. The concentrated and dialyzed supernatant fraction was precipitated four times $(\mathrm{v} / \mathrm{v})$ with $95 \%$ ethanol overnight at $4^{\circ} \mathrm{C}$. The precipitate was dissolved in deionized water. Deproteinization was carried out with the Sevag reagent, and this was repeated until there was no absorption at $280 \mathrm{~nm}$. The crude polysaccharide was purified by ultrafiltration (membrane MW cutoff: 5,000$)$ under pressure $(0.3 \mathrm{MPa})$ and lyophilized.

Artificial gastric and gastrointestinal fluid digestion. The operation process of artificial gastric fluid digestion was based on our previous studies (Lu et al., 2018). Digestion by artificial intestinal fluid was carried out as follows; the sample $(2.5 \mathrm{~g})$ was added to the artificial gastric medium and hydrolyzed at $37^{\circ} \mathrm{C}$ for $30 \mathrm{~min}$, then neutralized immediately by $\mathrm{NaOH}(1 \mathrm{M})$. Next, a phosphate buffer $\left(0.1 \mathrm{M}, \mathrm{pH}\right.$ 6.0), $\mathrm{NaHCO}_{3}$ $(1 \mathrm{M})$, amylase solution $(0.1 \mathrm{~mL}, 20 \mathrm{mg} / \mathrm{mL})$ and pancreatin solution $(5 \mathrm{~mL}, 25 \mathrm{mg} / \mathrm{mL})$ were added to the reaction system. The sample was hydrolyzed for $10,20,30,60,120,180$ and $240 \mathrm{mins}$ at $37^{\circ} \mathrm{C}$, followed by centrifugation $(10,000 \mathrm{rpm}, 10 \mathrm{~min})$ at $4^{\circ} \mathrm{C}$. The supernatant fraction was isolated by ultrafiltration (membrane MW cut-off: 5000) under pressure (0.3 MPa) and lyophilized to obtain AAPHs.

\section{Analysis of the degree of hydrolysis of AAPs with HPLC}

The AAPs hydrolyzates were performed by HPLC on an LC-20AD pump (Shimadzu, Japan) equipped with a TSK G3000 $\mathrm{SW}_{\mathrm{xL}}$ column $(7.8 \mathrm{~mm} \times 30 \mathrm{~cm})$, and a RID-10A refractometer (Shimadzu, Japan) as a detector. Elution was performed with a flow rate of $0.6 \mathrm{~mL} / \mathrm{min}$, the column was equilibrated with ultrapure water and elution was carried out with the same solution.

\section{Monosaccharide composition analysis with GC}

The monosaccharide compositions of AAPs and AAPHs were detected based on the previous study (Xu et al., 2016). Nine monosaccharides were standards, including xylose, 2-deoxy-D-glucose, arabinose, ribose, mannose, fucose, glucose, galactose and $\mathrm{N}$-acetyl-D-(+)-glucosamine.

\section{Purification of AAPHs}

AAPHs, dissolved in deionized water $(15 \mathrm{mg} / \mathrm{mL})$, was loaded onto a gel-filtration column $(3.5 \times 150 \mathrm{~cm})$ of Sephadex G-100 eluted with deionized water at a flow rate of $20 \mathrm{~mL} / \mathrm{h}$. The eluent fractions were collected and measured by the phenol-sulfuric acid method at $490 \mathrm{~nm}$. Three fractions were obtained, denoted as AAPHs1, AAPHs2 and AAPHs3. The corresponding fraction was concentrated, dialyzed and lyophilized, then re-dissolved in deionized water $(1.0 \mathrm{mg} / \mathrm{mL})$, and the UV absorption spectrum of the solution was recorded in the wavelength range of 190 500 nm.

\section{Molecular weight determination}

The $M_{w}$ of AAPHs1, AAPHs2 and AAPHs3 were determined by HPGPC. A Shimadzu apparatus equipped with a TSKgel G4000SW ${ }_{\mathrm{xL}}$ (TOSOH, Japan) column $(7.8 \times 300 \mathrm{~mm})$ and a RID were used. Ultrapure water was used as eluent, the column temperature was $33^{\circ} \mathrm{C}$, and $20 \mu \mathrm{L}$ of sample was injected after passing through a $0.22 \mu \mathrm{m}$ syringe filter. Six dextran stand$\operatorname{ards}\left(M_{w}=505,4300,20100,31200,158100\right.$ and $291600 \mathrm{Da})$ were applied for calibration. The standard curve represented the linear relationship of the retention time $\left(R_{T}\right)$ and the logarithm of the molecular weights. The $M_{w}$ of the sample was calculated using $R_{T}$ based on the equation of linear regression of the standard curve.

\section{Animals and their care}

Male Wistar rats (initial bw 200-250 g) were provided by the animal centre of Hangzhou Normal University. The animals were housed under conditions of $25 \pm 2{ }^{\circ} \mathrm{C}$ and relative humidity ranging from $50 \%$ to $70 \%$ with a $12 \mathrm{~h}$ darkness/light cycle and maintained with ad libitum access to water throughout the experimental period. The experiment was approved by the Committee for Laboratory Animal Care and Use of Hangzhou Normal University. All procedures were performed strictly following the "Guide for the care and use of laboratory animals" published by the National Institute of Health.

\section{Induction of T2DM in rats}

T2DM was induced in the rats by feeding them a highfat diet (HFD), which was prepared by mixing $20 \%$ sucrose $(\mathrm{w} / \mathrm{w}), 1 \%$ cholesterol $(\mathrm{w} / \mathrm{w}), 2.5 \%$ dried egg 
yolk powder and $10 \%$ lard fat $(\mathrm{w} / \mathrm{w})$ into the basal diet for 6 weeks, followed by intraperitoneal injection of STZ $(30 \mathrm{mg} / \mathrm{kg}$ bw) dissolved in $0.1 \mathrm{M}$ citrate buffer (pH 4.5). Two weeks later, the rats were confirmed to be in the diabetic stage with a fasting blood glucose higher than $15.6 \mathrm{mmol} / \mathrm{L}$, and were used as diabetic experiment animals in this study.

\section{EXPERIMENTAL DESIGN}

The continuous cycle of animal experiments were 4 weeks. The rats were randomly divided into five experimental groups $(n=5)$ as follow:

- group 1: the control group (C), normal rats administered with normal saline $(0.86 \% \mathrm{NaCl})$ by oral gavage at $5 \mathrm{ml} / \mathrm{kg}$ bw/day

- group 2: the diabetic model group (D), HFD and low-dose STZ induced diabetic rats administered with normal saline by oral gavage at $5 \mathrm{ml} / \mathrm{kg}$ bw/day

- group 3: the diabetic with STZ group (DS), HFD and low-dose STZ induced diabetic rats administered with acarbose in normal saline by oral gavage at $15 \mathrm{mg} / \mathrm{kg}$ bw/day

- group 4: the diabetic with STZ and APPs group (DSA), HFD and low-dose STZ induced diabetic rats administered with AAPs by oral gavage at $150 \mathrm{mg} / \mathrm{kg}$ bw/day. The dose of AAPs was chosen based on a previous study (Xu et al., 2016)

- group 5: the diabetic with STZ and AAPHs group (DSAH), HFD and low-dose STZ induced diabetic rats administered with AAPHs by oral gavage at $150 \mathrm{mg} / \mathrm{kg}$ bw/day.

\section{BIOCHEMICAL ANALYSIS}

The rats were fasted overnight and blood glucose levels in the tail vein were determined using a glucometer. Then fresh serum samples were prepared from the rat hearts and the serum insulin concentration was determined using an ELISA kit (QY-R2097). Enzyme activity of the antioxidant enzymes was determined using the test kits of CAT (A007-2), GR (A062), GSH (A006-2) and MDA (A003-1). The liver was homogenized and centrifuged for $15 \mathrm{~min}$ at $5000 \mathrm{rpm}$. The supernatant was obtained for the determination of hepatic glycogen by anthrone spectrophotometric method (Agbagla-Dohnani et al., 2012).

\section{Determination of GLP-1 secretion level}

GLP-1 secretion level was determined at the end of the experiment. After $18 \mathrm{~h}$ fasting, the rats in different groups were administered with $5 \mathrm{ml}$ of normal saline, $15 \mathrm{mg} / \mathrm{kg}$ bw of acarbose and $150 \mathrm{mg} / \mathrm{kg}$ bw of AAPs and AAPHs, successively. After $1 \mathrm{~h}$, the rats were orally gavaged with $2 \mathrm{~g} / \mathrm{kg}$ bw of glucose dissolved in deionized water. Then, blood samples from the eyeball vein were collected at $0,30,60$ and 120 min after glucose loading under light ether anesthesia. The serum GLP-1 concentration was determined using a commercial rat kit GLP-1 ELISA (QY-R2115).

\section{STATISTICAL ANALYSIS}

All data were shown as means \pm standard deviations and carried out in triplicate. ANOVA was used for multiple comparisons, and $P$ values of less than 0.05 were considered statistically significant.

\section{RESULTS AND DISCUSSION}

\section{Simulated digestion of AAPs in vitro}

Based on our previous study (Lu et al., 2018), AAPs could be degraded to some extent in the artificial gastric fluid in vitro, and gastric acid and enzymes had a certain influence on AAPs. Thus, after 30 minutes of digestion in an artificial gastric fluid, AAPs were further digested in the artificial intestinal fluid. As shown in Table 1, there was no difference in retention time in

Table 1. $R_{T}$ for $A$. auricula polysaccharides after artificial simulated intestinal digestion in vitro

\begin{tabular}{cccc}
\hline $\begin{array}{c}\text { Hydrolysis } \\
\text { reaction time } \\
\text { min }\end{array}$ & \multicolumn{3}{c}{ Retention time of main peak } \\
min \\
10 & 12.31 & 16.55 & 19.58 \\
20 & 12.24 & 16.48 & 19.56 \\
30 & 12.30 & 16.58 & 19.54 \\
60 & 12.28 & 16.54 & 19.25 \\
120 & 12.29 & 16.56 & 19.54 \\
180 & 12.30 & 16.51 & 19.57 \\
210 & 12.30 & 16.57 & 19.57 \\
\hline
\end{tabular}



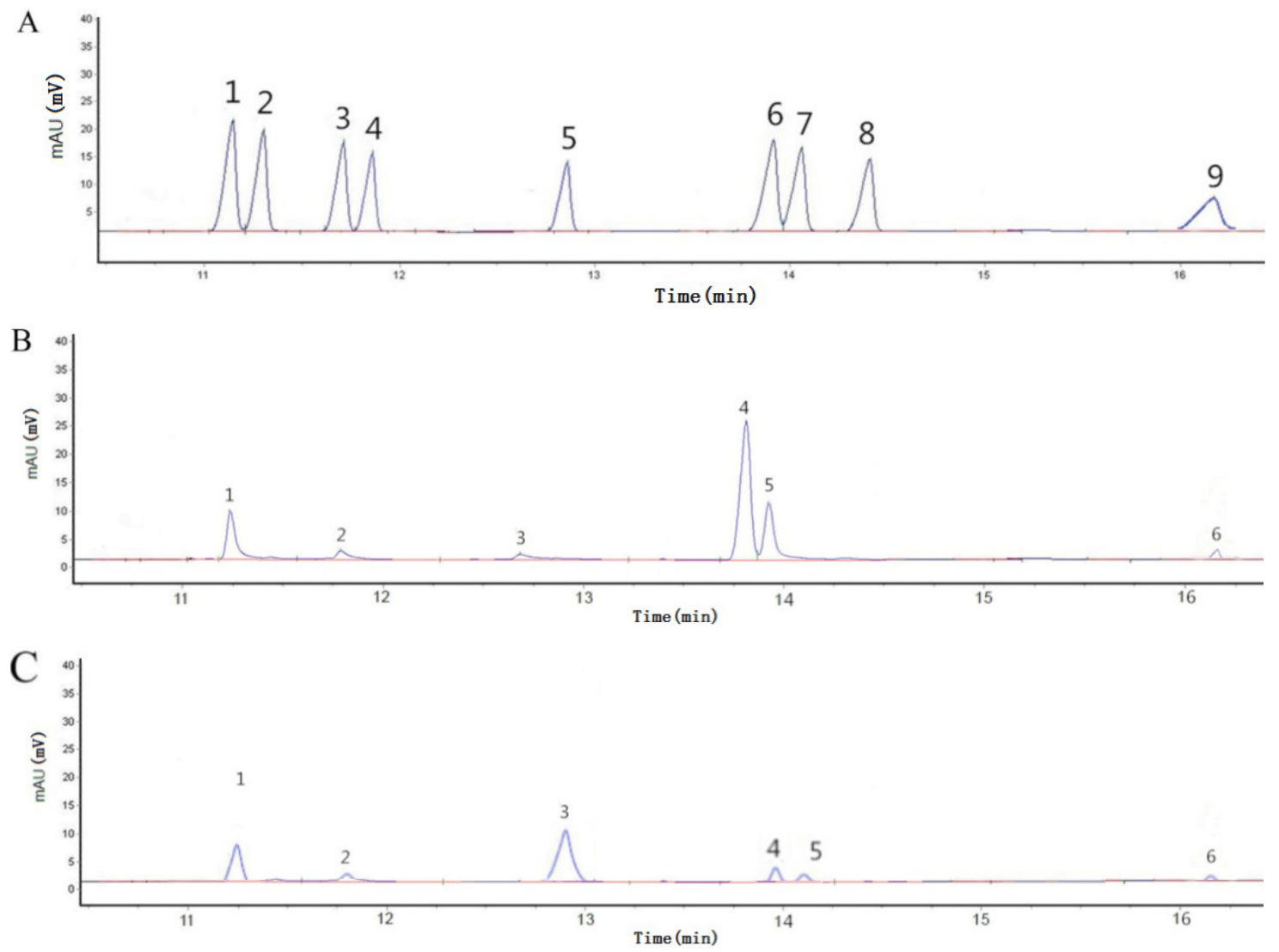

Fig. 1. GC spectrum: A - standard monosaccharide mixture; 1 - ribose, 2 - arabinose, 3 - xylose, 4 - fucose, 5 - 2-deoxy-D-glucose, 6 - mannose, 7 - glucose, 8 - galactose, 9 - N-acetyl-D-(+)-glucosamine; B - AAPs; C - AAPHs

the artificial intestinal juice at different time periods. As hydrolysis time went on, the chromatographic peak number and the peak time remained unchanged. The results showed that AAPs could be degraded in artificial gastric fluid in vitro, but could not be degraded in artificial intestinal fluid. Thus, the hydrolysates (AAPHs) were prepared after $30 \mathrm{~min}$ of digestion in artificial gastric juice and used in the following experiments.

\section{Molecular composition analysis}

The molecular composition of A. auricula polysaccharides (AAPs) and their hydrolyzed product (AAPHs) was analyzed using gas chromatograms (Fig. 1).

AAPs presented four major components with arabinose, mannose, glucose and glucosamine, and mannose was the predominant sugar in AAPs. The difference was that AAPHs presented four major components with arabinose, 2-deoxy-glucose, mannose and glucose, and 2-deoxy-glucose was the predominant sugar in AAPHs. It was inferred that AAPs was a kind of heteropolysaccharide, and contained arabinose, xylose, 2-deoxy-glucose, mannose, glucose, and glucosamine with the molar ratio of $1: 0.44: 0.33: 1.67$ $: 1: 0.17$. AAPHs also was a kind of heteropolysaccharide, and contained arabinose, xylose, 2-deoxyglucose, mannose, glucose and glucosamine with the molar ratio of $2.85: 0.78: 4.31: 1.49: 1.00: 0.34$.

\section{Purification of AAPHs}

Gel-filtration chromatography was performed for the purification of AAPHs, and the curve of elution 


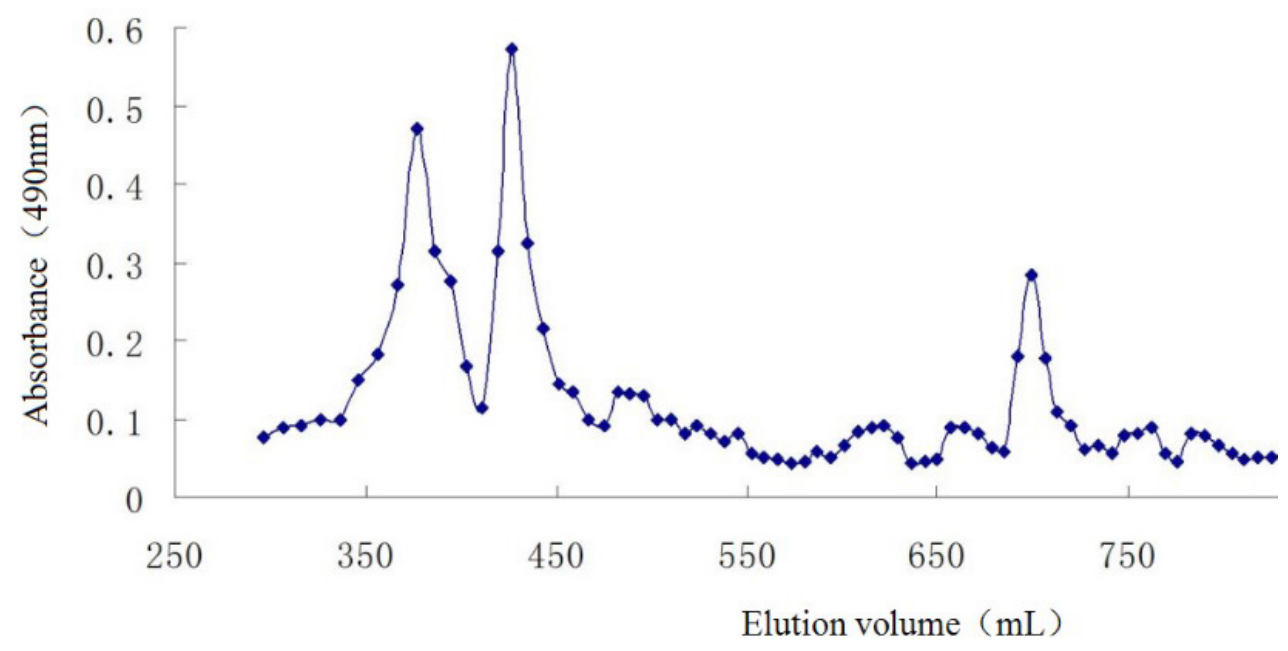

Fig. 2. The profile of AAPHs eluted from a Sephadex G-100 column $(3.5 \times 150 \mathrm{~cm})$
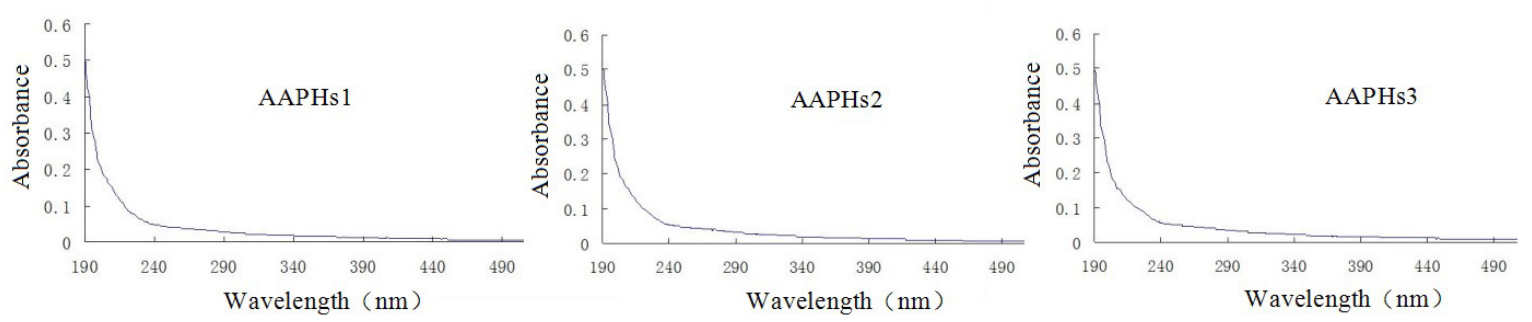

Fig. 3. UV spectrum of AAPHs1, AAPHs2 and AAPHs3

was shown in Figure 2. Three fractions (AAPHs1, AAPHs2 and AAPHs3) were obtained from a Sephadex G-100 column. In addition, no significant absorbance was observed at $260 \mathrm{~nm}$ or $280 \mathrm{~nm}$ in the UV spectrum (Fig. 3), suggesting that the three fractions did not contain protein or nucleic acid.

\section{The molecular weight of AAPHs1, AAPHs2 and AAPHs3}

The average molecular weights of AAPHs1, AAPHs2 and AAPHs3 were determined by HPLC. Dextrans with different molecular weights were used for preparing a standard curve. Figure 4 shows the standard equation $\log M_{w}=-0.4496 T_{R}+10.734\left(R^{2}=0.9972\right)$ and its curve. AAPHs1, AAPHs2 and AAPHs3 were eluted as a single symmetrical peak, as determined by HPGPC in Figure 5, which showed that AAPHs1,
AAPHs2 and AAPHs3 were homogeneous and purified. The molecular weights of AAPHs1, AAPHs2 and AAPHs3 were found to be $320,169,62 \mathrm{kDa}$ respectively, according to the calibration curve.

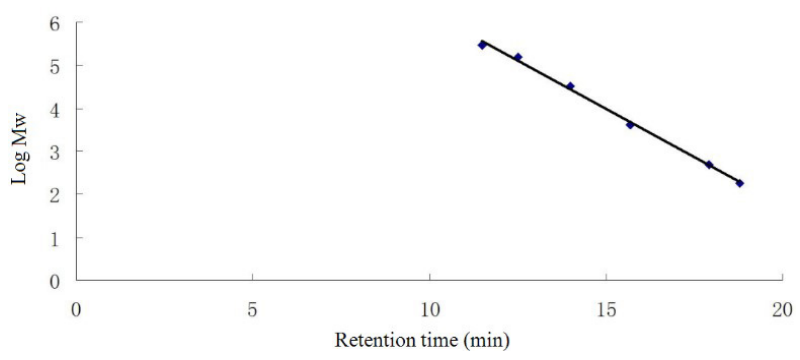

Fig. 4. Standard curve of determining the molecular weight 

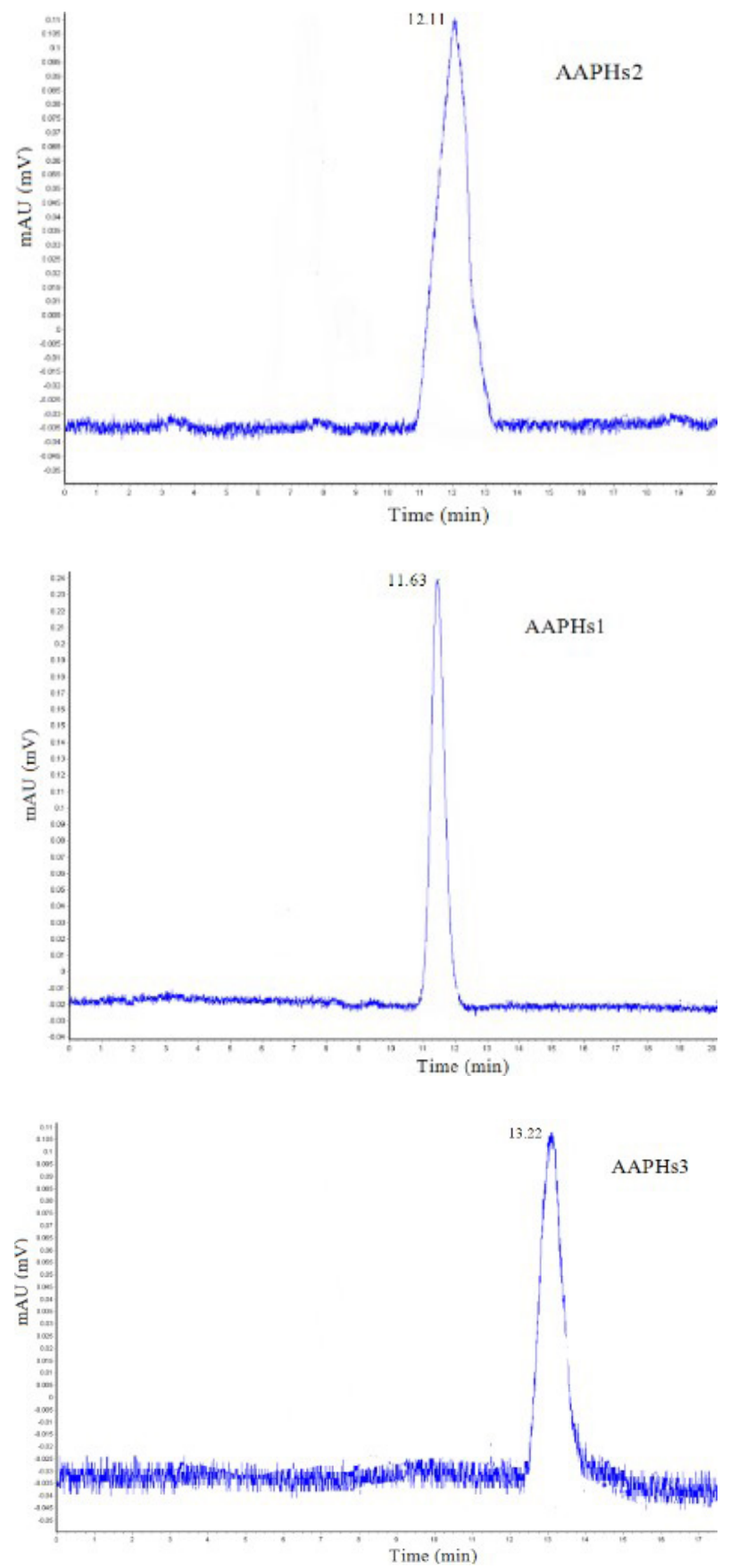

Fig. 5. HPGPC profile of AAPHs1, AAPHs2 and AAPHs3

\section{Changes in enzyme activities and antioxidant}

The results of the antioxidant enzyme activities (CAT and GR), as well as GSH and MAD contents, were shown in Figure 6.
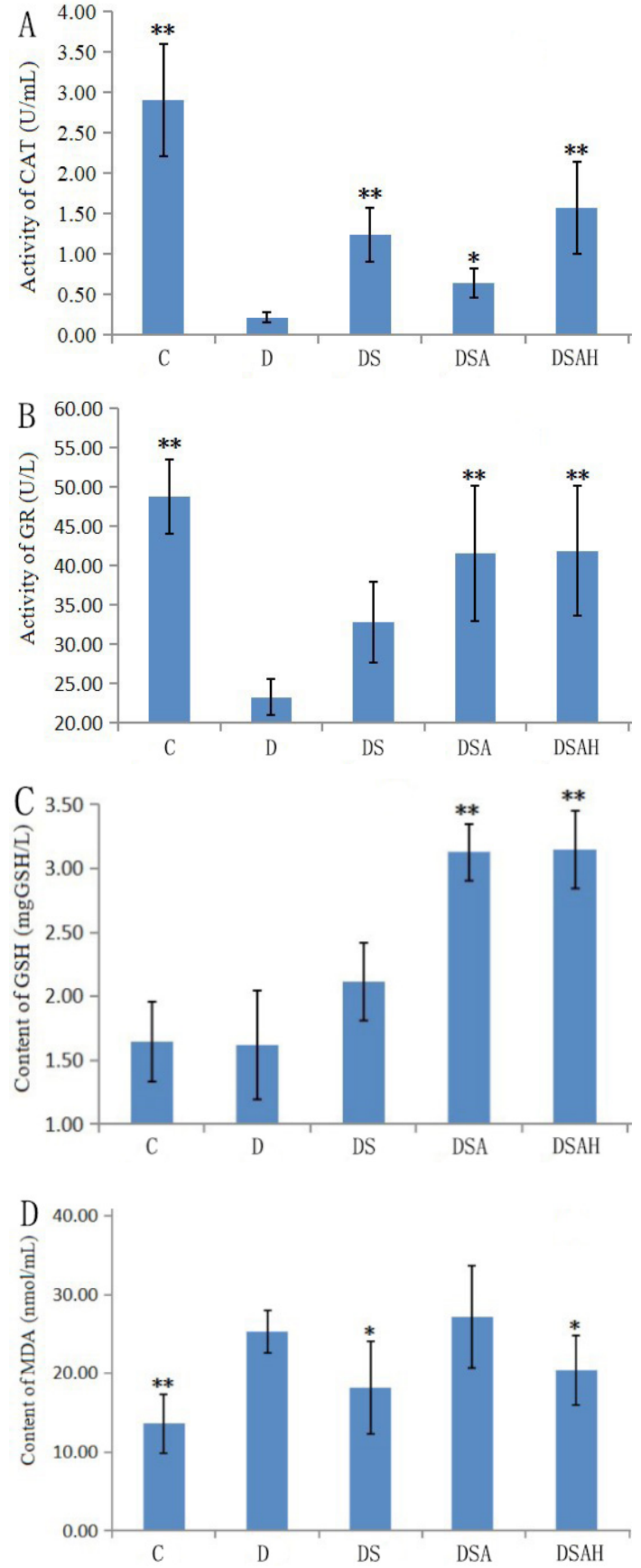

Fig. 6. Effects of AAPs and AAPHs on the activities of antioxidant enzymes and the level of GSH and MDA in the serum of T2DM rats: A - the activity of CAT, B - activity of GR, C - level of GSH, D - level of MDA. All data is presented as means $\pm \mathrm{SD}(n=5) .{ }^{*} p<0.05$ - significant from the diabetic model (D). ${ }^{* *} p<0.01-$ significant from the diabetic model (D) 
AAPHs displayed an extraordinary ability to enhance the activities of GR and CAT, as well as the level of GSH, while reducing the content of MDA compared with the diabetic model group (D). The activity of CAT for AAPHs and AAPs was $1.57 \pm 0.57$ $\mathrm{U} / \mathrm{mL}$ and $0.64 \pm 0.18 \mathrm{U} / \mathrm{mL}$, respectively, which was significantly higher than that of diabetic model group (D) $(0.22 \pm 0.07 \mathrm{U} / \mathrm{mL}, P<0.01$ and $P<0.05)$. The activity of GR was $41.87 \pm 6.25 \mathrm{U} / \mathrm{L}$ and $41.59 \pm 6.57$ $\mathrm{U} / \mathrm{L}$ for AAPHs and AAPs respectively, which was significantly higher than that of the diabetic model group (D) $(23.31 \pm 2.26 \mathrm{U} / \mathrm{L}, P<0.01$ and $P<0.01)$. In addition, AAPHs and AAPs also possessed a remarkable capability to increase the level of GSH. The level of GSH reached $3.14 \pm 0.30 \mathrm{mg} \mathrm{GSH} / \mathrm{L}$ and 3.13 $\pm 0.22 \mathrm{mg} \mathrm{GSH} / \mathrm{L}$ for AAPHs and AAPs respectively, which was significantly higher than that of the diabetic model group (D) $(1.62 \pm 0.42 \mathrm{mg} \mathrm{GSH} / \mathrm{L}, P<0.01$ and $P<0.01)$. Additionally, AAPHs caused a significant decrease in the level of MDA $(18.57 \pm 1.67 \mathrm{nmol} / \mathrm{mL}$, $P<0.05)$ compared with that of the diabetic model group (D) $(25.30 \pm 2.69 \mathrm{nmol} / \mathrm{mL})$, while AAPs had no effect on MDA in this study.

\section{Changes in FBG, glycogen, C-peptide and insulin levels in T2DM rats}

HFD could play an important role in inducing insulin resistance while low-dose STZ can partially damage islet cells, similar to in T2DM in humans (Jiao et al., 2017), and the integration of HFD and low-dose STZ substituted as an animal model to similar the human T2DM. As expected, the rats administered HFD and a low dose of STZ had significantly elevated fasting blood glucose (FBG), and evident damage to islet and hepatic cells. A rat model of type 2 diabetes mellitus was successfully established.

As shown in Table 2, the FBG level of the control group (C) remained constant throughout the experimental period, and six weeks of high-fat diet (HFD) feeding and 2 weeks with low-dose STZ brought about a 4 -fold and $23.7 \%$ increase in blood glucose levels compared to the control group (C) respectively, and there was a significant difference compared to the diabetic model rats $(\mathrm{D})(P<0.05)$.

\section{Effect on GLP-1 secretion in vivo}

The effect of AAPs and AAPHs on GLP-1 secretion for experimental type 2 diabetic rats was evaluated.

As shown in Figure 7, the glucose-stimulated GLP-1 secretion in a diabetic rat was impaired and exhibited a reduction after oral glucose administration during the experimental period compared to the control group (C). The AAPHs group showed a significant increase in GLP-1 level $(P<0.05)$ compared to the diabetic model group (D) from $0 \mathrm{~min}$ to $30 \mathrm{~min}$, but no significant difference was observed at $60 \mathrm{~min}$. For the AAPs group, there was no significant difference compared to the diabetic model group (D) during the experimental period.

Table 2. The effects of AAPs and AAPHs on FBG, glycogen, C-peptide and insulin level in T2DM rats

\begin{tabular}{lccccc}
\hline \multirow{2}{*}{ Group } & \multicolumn{2}{c}{ FBG levels, mmol/L } & Liver glycogen \\
\cline { 2 - 4 } & $\begin{array}{c}\text { beginning of } \\
\text { experiment }\end{array}$ & end of experiment & & $\begin{array}{c}\text { Plasma C-peptide } \\
\text { ng/L }\end{array}$ & $\begin{array}{c}\text { Plasma insulin } \\
\text { mIU/L }\end{array}$ \\
\hline C & $4.88 \pm 0.22^{* *}$ & $4.40 \pm 0.47^{* *}$ & $48.33 \pm 2.05^{* *}$ & $41.48 \pm 9.36^{* *}$ & $1.28 \pm 0.35^{*}$ \\
D & $19.54 \pm 2.38$ & $18.64 \pm 5.67$ & $40.33 \pm 2.51$ & $23.91 \pm 2.51$ & $0.44 \pm 0.12$ \\
DS & $18.94 \pm 2.23$ & $11.78 \pm 2.77^{*}$ & $47.99 \pm 1.17^{*}$ & $31.58 \pm 9.94^{*}$ & $1.19 \pm 0.21^{*}$ \\
DSA & $19.16 \pm 1.29$ & $13.80 \pm 3.03^{*}$ & $46.32 \pm 4.56^{*}$ & $34.67 \pm 13.22^{*}$ & $0.51 \pm 0.10$ \\
DSAH & $19.20 \pm 1.56$ & $14.22 \pm 5.04^{*}$ & $44.81 \pm 1.82^{*}$ & $29.14 \pm 1.86^{*}$ & $0.55 \pm 0.11$ \\
\hline
\end{tabular}

Data presented as means $\pm \mathrm{SD}(n=5) .{ }^{*} p<0.05$ significant from the diabetic control (D), ${ }^{* *} p<0.01$ significant from the diabetic control (D). 


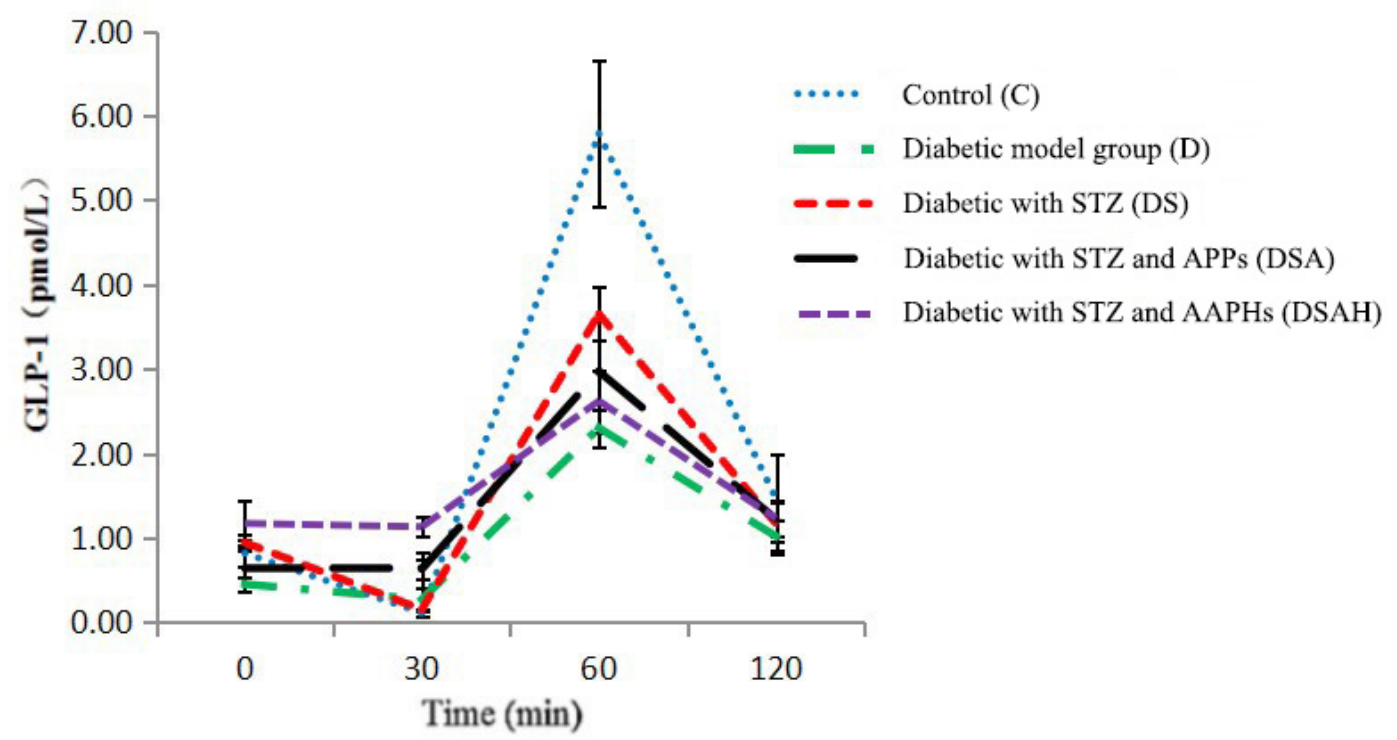

Fig. 7. Effect of AAPs and AAPHs on GLP-1 levels of T2DM rats. Values are expressed as means \pm SD $(n=5)$

\section{DISCUSSION}

A. auricula polysaccharides could be degraded in the environment of artificial gastric fluid, but could not be degraded in the artificial intestinal fluid in vitro. The hydrolyzed product (AAPHs) from AAPs was composed of three fractions and the molecular weight of these three fractions were the difference. AAPHs exhibited marked antioxidative capabilities compared with the diabetic model rats. One of the main pathological mechanisms suggested in diabetes is the oxidative stress caused by the production of excess reactive oxygen species (ROS; Tiwari et al., 2013). It is commonly known that oxidative stress arises under the conditions of imbalance between free radical release and the antioxidant potential of non-enzymatic and enzymatic substances in tissues (Rumyana et al., 2016), so it is vital to enhance the antioxidant capability for relieving oxidative stress. Glutathione (GSH), an important antioxidant in the body, and the primary antioxidant enzymes for catalase (CAT) and glutathione reductase (GR), can effectively inhibit free radical generation and improve oxidative damage in cells or organisms.

In the present study, the induced hyperglycemia in the rats became more pronounced with increasing production of MDA and decreasing non-enzymatic (GSH) and enzymatic (CAT, GR) antioxidant defense (Fig. 6). These are the cytotoxic effects of STZ that can be partially mediated through the formation of NO and ROS, which is explained by Takasu et al. (1991) in their studies. The results demonstrated that AAPHs had marked potential to enhance the activities of CAT (Fig. 6A) and GR (Fig. 6B) in rat serum compared to diabetic model rats, which showed that AAPHs possesses remarkable antioxidant activity in vivo. GSH, an important antioxidant in the body, can scavenge free radicals and plays an important part in the biochemical defense system of the body. CAT is a key enzyme of the antioxidant defense system and can break down $\mathrm{H}_{2} \mathrm{O}_{2}$ into water, and GR plays a key role in the scavenging of ROS during oxidative stress. Hence, the enhanced level of GSH and activities of these enzymes may provide an effective defense to free radical damage. Malondialdehyde (MDA), an important by-product of lipid peroxidation, demonstrates the degree of lipid peroxidation (Zeng et al., 2017). As shown in Figure 6D, the MDA level was reduced in AAPHs groups, which suggested that certain active groups were produced or exposed after the hydrolysis of AAPs and had the ability to inhibit the production of cytotoxic chemicals. 
The liver can regulate the amount of blood glucose in the body. However, in diabetes, glycogen output has a positive correlation with fasting blood glucose, which is the primary cause for the hyperglycemia. As shown in Table 2, the liver glycogen level of AAPHs (diabetes + AAPHs) and AAPs (diabetes + AAPs) increased significantly $(P<0.05)$ compared with the diabetic model group (D). This suggested that both AAPHs and AAPs could improve glucose metabolism, inhibiting hepatic glucose output, and raising glycogen content. Therefore, AAPs and their simulated hydrolysate might both exhibit a certain therapeutic effect in the liver function to HFD and low-dose STZ-induced T2DM.

Testing plasma insulin and C-peptide levels is helpful in assessing insulin resistance and predicting the progress of T2DM. In the present study, C-peptide and insulin secretory capacity in plasma was significantly lower in the diabetic model rats $(p<0.05)$ compared to the control (normal) rats. There was a significant difference in C-peptide level $(p<0.05)$ between the AAPHs (diabetes + AAPHs) and AAPs (diabetes + AAPs) groups and the diabetic model group (D), but treatment with AAPHs and AAPs did not affect the insulin level in diabetic rats. This was perhaps due to the different biological half-lives of insulin and $\mathrm{C}$ peptide (the halflife of insulin is $4.8 \mathrm{~min}$, and of C peptide $11 \mathrm{~min}$ ). Serum insulin content is partly dependent on body mass and the degree of insulin resistance, while C-peptide reflects islet $\beta$ cell reserves and may offer a more accurate measurement of $\beta$ cell insulin response to glucose (Mallipedhi et al., 2015). Therefore, this data suggests that AAPs and their simulated hydrolysate might relieve hyperglycemia by improving islet function.

\section{ACCURATE MEASUREMENT}

AAPs have been reported to have evident antioxidant properties and anti-diabetic effects and prevent oxidative stress (Xu et al., 2016; Yuan et al., 1998; Zhang et al., 2011). It has been reported that some oligosaccharides can exhibit marked anti-diabetic effects in experimental T2DM (Li et al., 2012). The present study demonstrated that Auricularia auricular polysaccharides, as well as their hydrolysis, could ameliorate impaired islet function and improve the hepatic injury brought on by the experimental T2DM to some extent.
GLP-1 can exert its effects through the promotion of the proliferation of $\beta$-cells, and the inhibition of the $\beta$-cells apoptosis. Moreover, endogenous GLP-1 secretion by direct stimulation, or endogenous GLP-1 generation by elimination of the factors resulting in its attenuation, may be a viable method to strengthen GLP-1 action (Lai et al., 2017). Our findings indicated that AAPHs could partly restore the STZ-induced damage of GLP-1 secretion, inhibit the oxidative stress pathway, and slow down the apoptosis of $\beta$-cells. In addition, the effect of the hydrolysates from $A$. auricula polysaccharides was better than the polysaccharides themselves.

\section{CONCLUSIONS}

In the present study, Auricularia auricular polysaccharide simulated hydrolysates (AAPHs) were prepared from Auricularia auricular polysaccharides (AAPs) throughout hydrolysis by artificial gastrointestinal fluid. AAPs and AAPHs are both heteropolysaccharides and comprised of six monosaccharide components (arabinose, xylose, 2-deoxy-glucose, mannose, glucose and glucosamine at different molar ratios). AAPHs consisted of three components, and the molecular weights of the three components were 320 , $169,62 \mathrm{kDa}$ respectively. Additionally, evaluation of the antioxidant and anti-diabetic activities in vivo of AAPs and AAPHs was performed. Using HFD and low-dose STZ-induced Wistar rats as a T2DM model, administration of AAPHs appears to be better than AAPs in terms of antioxidant activities. AAPHs exhibited an extraordinary ability to enhance the activities of antioxidant enzymes (GR and CAT) and the level of $\mathrm{GSH}$, and to reduce the level of MDA compared to the diabetic model group (D). Meanwhile, AAPHs could significantly increase FBG, liver glycogen and plasma C-peptide levels in experimental type 2 diabetic rats.

\section{REFERENCES}

Agbagla-Dohnani, A., Cornu, A., Broudiscou, L. P. (2012). Rumen digestion of rice straw structural polysaccharides: Effect of ammonia treatment and lucerne extract supplementation in vitro. Animal, 6(10), 1642-1647. http://dx.doi.org/10.1017/S175173111200050X 
Lu, A., Yu, M., Shen, M., Fang, Z., Xu, Y., Wang, S., Zhang, Y., Wang, W. (2018). Antioxidant and anti-diabetic effects of Auricularia auricular polysaccharides and their degradation by artificial gastrointestinal digestion - Bioactivity of Auricularia auricular polysaccharides and their hydrolysates. Acta Sci. Pol. Technol. Aliment., 17(3), 277-288. http://dx.doi.org/10.17306/J.AFS.2018.0557

Chapman, A., Meyer, C., Renehan, E., Hill, K. D., Browning, C. J. (2017). Exercise interventions for the improvement of falls-related outcomes among older adults with diabetes mellitus: A systematic review and metaanalyses. J. Diab. Compl., 31, 631-645. http://dx.doi. org/10.1016/j.jdiacomp.2016.09.015

Chiu, W. C., Yang, H. H., Chiang, S. C., Chou, Y. X., Yang, H. T. (2014). Auricularia polytricha aqueous extract supplementation decreases hepatic lipid accumulation and improves antioxidative status in animal model of nonalcoholic fatty liver. Biomedicine, 4, 29-38. http://dx.doi. org/10.7603/s40681-014-0012-3

Daisy, F., Mayyada, E., Dalia, R. (2015). Evaluation of the antioxidant activity of enzymatically-hydrolyzed sulfated polysaccharides extracted from red algae; Pterocladia capillacea. LWT - Food Sci. Techn., 63, 1236-1244. http://dx.doi.org/10.1016/j.lwt.2015.04.024

Jiao, Y. K., Zhang, M. L., Wang, S. M., Yan, C. Y. (2017). Consumption of guava may have beneficial effects in type 2 diabetes: A bioactive perspective. Int. J. Biol. Macromol., 101, 543-552. http://dx.doi.org/10.1016/j. ijbiomac.2017.03.130

Kim, S. K., Hong, U. P., Kim, J. S., Kim, C. H., Lee, K. W., Choi, S.E., ..., Lee, M. W. (2007). Anti-diabetic effect of Auricularia auricula mycelia in streptozotocin-induced diabetic rats. Natural Prod. Sci., 13(4), 390-393.

Lai, F. H., Chen, Y., Lin, H. M., Wang, X. L., Zhu, X. M., Li, Y. B., ..., Cao, X. P. (2017). Pancreatic-derived factor impaired glucagon-like Peptide-1 production from GLUTag enterendorine L-cell line and intestines. Molec. Cell. Endocrinol., 452(C), 110-119. http:// dx.doi.org/10.1016/j.mce.2017.05.021

Lau, D. C. (2014). Canada welcomes a novel class of oral glucose-lowering drugs for people with Type 2 diabetes. Can. J. Diab., 38(4), 219-220. http://dx.doi. org/10.1016/j.jcjd.2014.06.001

Li, P. B., Lin, W. L., Wang, Y. G., Peng, W., Cai, X. Y., Su, W. W. (2012). Anti-diabetic activities of oligosaccharides of Ophiopogonis japonicus in experimental type 2 diabetic rats. Int. J. Biol. Macromol., 51, 749-755. http://dx.doi.org/10.1016/j.ijbiomac.2012.07.007

Liu, M., Liu, Z., Xu, B., Zhang, W., Cai, J. (2016). Review of systematic reviews and Meta-analyses investigating Traditional Chinese Medicine treatment for type 2 diabetes mellitus. JTCM - J. Trad. Chinese Med., 36, 555563. http://dx.doi.org/10.1016/S0254-6272(16)30074-7

Lu, A. X., Yu, M. E., Shen, M., Xu, S. Q., Xu, Z. Q., Zhang, Y. J., ..., Wang, W. M. (2018). Preparation of the $A u$ ricularia auricular polysaccharides simulated hydrolysates and their hypoglycaemic effect. Int. J. Biol.
Macromol., 106, 1139-1145. http://dx.doi.org/10.1016/j. ijbiomac.2017.08.118

Mallipedhi, A., Min, T., Prior, S. L., MacIver, C., Luzio, S. D., ..., Stephens, J. W. (2015). Association between the preoperative fasting and postprandial C-peptide AUC with resolution of type 2 diabetes 6 months following bariatric surgery. Metabolism, 64, 1556-1563. http:// dx.doi.org/10.1016/j.metabol.2015.08.009

Mizuno, T., Saito, H., Nishitoba, T., Kawagishi, H. (1995). Antitumor active substances from mushrooms. Food Rev. Int., 11, 23-61.

Nguyen, T. L., Wang, D. Y., Hu, Y. L., Fan, Y. P., Wang, J. M., ..., Dang, B. K. (2012). Immuno-enhancing activity of sulfated Auricularia auricula polysaccharides. Carbohydr. Polym., 89, 1117-1122. http://dx.doi. org/10.1016/j.carbpol.2012.03.082

Qiu, J., Zhang, H., Wang, Z., Liu, S., Regenstein, J. M. (2016). Response surface methodology for the synthesis of an Auricularia auricula judae polysaccharides-CDDP complex. Int. J. Biol. Macromol., 93, 333-343. http:// dx.doi.org/10.1016/j.ijbiomac.2016.06.066

Rumyana, S., Vessela, V., Ilina, K., Petranka, Z., Spiro, K., Iliana, I. (2016). Anti-diabetic and antioxidant effects of saponarin from Gypsophila trichotoma on streptozotocin-induced diabetic normotensive and hypertensive rats. Phytomedicine, 23, 483-490. http://dx.doi. org/10.1016/j.phymed.2016.02.024

Suphavadee, C., Pornpan, S., Natamart, K., Suttaya, S. (2016). Oligosaccharide production from agricultural residues by nonstarch polysaccharide degrading enzymes and their prebiotic properties. Agric. Agricult. Sci. Proc., 11, 131-136. http://dx.doi.org/10.1016/j.aaspro. 2016.12.022

Takasu, N., Komiya, I., Asawa, T. (1991). STZ and alloxaninduced $\mathrm{H}_{2} \mathrm{O}_{2}$ generation and DNA fragmentation in pancreatic islets. Diabetes, 40, 1141-1145. http://dx.doi. org/10.2337/diabetes.40.9.1141

Tiwari, B. K., Pandey, K. B., Abidi, A. B., Rizvi, S. I. (2013). Markers of oxidative stress during diabetes mellitus. J. Biomark., 1, 1-8. http://dx.doi.org/10.1155/2013/378790

Wang, L. C., Wu, H., Ji, J., Xue, F., Liu, R. (2016). Preparation, analysis and antioxidant evaluation of the controlled product of polysaccharide from Mactra veneriformis by mild acid hydrolysis. Carbohydr. Polym., 137, 709-718. http://dx.doi.org/10.1016/j.carbpol.2015.11.030

Wang, T., Xu, Q., Wu, Y., Zeng, A., Li, M., Gao, H. (2009). Quaternized chitosan (QCS)/poly (aspartic acid) nanoparticles as a protein drug-delivery system. Carbohydr. Res., 344, 908-914. http://dx.doi.org/10.1016/j. carres.2009.02.018 
Wu, Q., Tan, Z. P., Liu, H. D., Gao, L., Wu, S. J., Luo, J. W., ..., Xu, X. H. (2010). Chemical characterization of Auricularia auricula polysaccharides and its pharmacological effect on heart antioxidant enzyme activities and left ventricular function in aged rats. Int. J. Biol. Macromol., 46, 284-288. http://dx.doi.org/10.1016/j. ijbiomac.2010.01.016

Xiong, W., Zhang, Q. T., Yin, F., Yu, S. H., Ye, T. T., Pan, W. S., Yang, X. G. (2016). Auricularia auricular polysaccharide low molecular weight chitosan polyelectrolyte complex nanoparticles: Preparation and characterization. Asian J. Pharm. Sci., 11, 439-448. http://dx.doi. org/10.1016/j.ajps.2015.10.064

Xu, J., Xu, L. L., Zhou, Q. W., Hao, S. X., Zhou, T., Xie, H. J. (2015). Isolation, purification, and antioxidant activities of degraded polysaccharides from Enteromorpha prolifera. Int. J. Biol. Macromol., 81, 1026-1030. http:// dx.doi.org/10.1016/j.ijbiomac.2015.09.055

Xu, S. Q., He, J. M., Wang, S., Zhang, Y. J., Zhu, L. Y., Wang, G., Wang, W. M. (2016). Studies on molecular composition and functional properties of the non-starch polysaccharide from Auricularia auricula. J. Chinese Inst. Food Sci. Technol., 16, 65-70. http://dx.doi.org/1 $0.16429 /$ j.1009-7848.2016.08.010
Xu, S. Q., Zhang, Y. J., Jiang, K. (2016). Antioxidant activity in vitro and in vivo of the polysaccharides from different varieties of Auricularia auricula. Food Funct., 7(9), 3868-3879. http://dx.doi.org/10.1039/C6FO00686H

Yoon, S. J., Yu, M. A., Pyun, Y. R., Hwang, J. K., Chu, D. C. (2003). The nontoxic mushroom Auricularia auricula contains a polysaccharide with anticoagulant activity mediated by anti-thrombin. Thromb. Res., 112, 151158. http://dx.doi.org/10.1016/j.thromres.2003.10.022

Yuan, Z. M., He, P. M., Cui, J. H., Takeuchi, H. (1998). Hypoglycemic effect of watersoluble polysaccharide from Auricularia auricula-judae Que. on genetically diabetic KK-Ay rats. Biosci. Biotechn. Biochem., 62, 18981903. http://dx.doi.org/10.1271/bbb.62.1898

Zeng, W. C., Sun, Q., Zhang, W. H., Liao, X. P., Shi, B. (2017). Antioxidant activity in vivo and biological safety evaluation of a novel antioxidant peptide from bovine hair hydrolysates. Process Biochem., 56, 193-198. http://dx.doi.org/10.1016/j.procbio.2017.02.022

Zhang, H., Wang, Z. Y., Zhang, Z., Wang, X. (2011). Purified Auricularia auricular-judae polysaccharide (AAP I-a) prevents oxidative stress in an ageing mouse model. Carbohydr. Polym., 84(1), 638-648. http://dx.doi. org/10.1016/j.carbpol.2010.12.044 